\title{
New Presentation of the Acupuncture Law of Five Elements Considering the Shape of the Human NEMF
}

\author{
Maria Kuman* \\ Holistic Research Institute, USA \\ *Corresponding author: Maria Kuman, Holistic Research Institute, 1414 Barcelona Dr, Knoxville, USA.
}

To Cite This Article: Maria Kuman. New Presentation of the Acupuncture Law of Five Elements Considering the Shape of the Human NEMF. Am J Biomed Sci \& Res. 2019 - 5(3). AJBSR.MS.ID.000903. DOI: 10.34297/AJBSR.2019.05.000903.

Received: 鮆September 01, 2019; Published: 眥 September 18, 2019

\begin{abstract}
The law of five elements in ancient acupuncture reflects the order, in which the organs in the body become active. When acupuncture treatments are done this needs to be taken into consideration. This article discusses the Quantum Computer in the Subconscious, which works with the waves of our torus shaped NEMF, and through the waves of NEMF rules and regulates all the organs in the body. Based on this, this article offers a new presentation of the ancient acupuncture law of five elements, which consider the shape of the human nonlinear electromagnetic field (NEMF). The torus shape of the human NEMF and its dynamic was presented as two intersecting pyramids one with top up, the other with top down, in dynamic equilibrium. The upsidedown Yang pyramid represents the five hollow (Yang) organs, which are more active (Yang=active). The upright pyramid represents the five solid (Yin) organs, which are less active (Yin=passive). Then the coupled organs with strong functional dependence are on the vertical lines connecting the bases of the two pyramids and the vertical line connecting the two tops.
\end{abstract}

Keywords: Acupuncture practice; Law of five elements; The acupuncture laws and the NEMF; NEMF and the Quantum Computer

\section{Introduction}

The author presented in 1983 at the 8th World Congress of Acupuncture nonlinear mathematical model of one acupuncture meridian [1]. The nonlinear equation had two types of solutions electric impulse and wave. The Chinese measured electric impulse running from the treated acupuncture point in direction of the acupuncture meridian, but nobody has detected waves. The author insisted wave must exist. Intrigued, a Hungarian scientist [2] started research for finding the waves. One year later he reported that he found that waves are running along the acupuncture meridians all the time, but when an acupuncture point of the meridian is treated with acupuncture, wave is generated which modifies the constantly running wave.

The waves running along the acupuncture meridians belong to the weak nonlinear electromagnetic field (NEMF) of the human body, which is informational in character, and from the subconscious rules and regulates everything in the body. The waves of this field are the basis of the operating in the Subconscious Quantum Computer, which rules and regulates all organs on holographic principle through the waves of the NEMF [3]. (We know this from the fact that the acupuncture points of the whole body are holographically represented on the ears, the palms, the feet, and the irises of the eyes [3]).

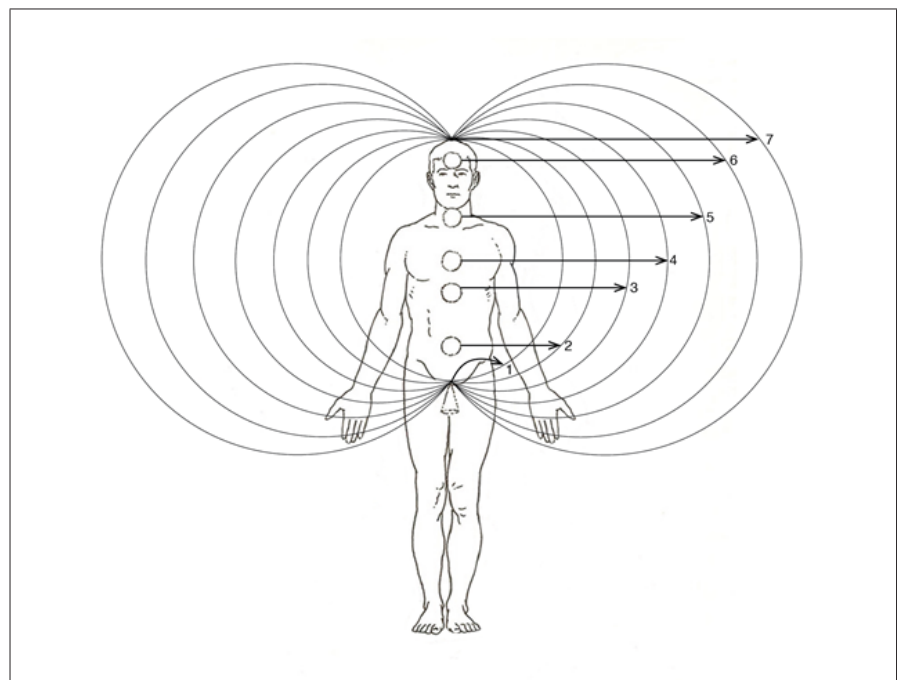

Figure 1: Vertical cross-section of the male NEMF with the chain of alternating vortices and anti-vortices along the backbone and the corresponding to them quantum energy levels.

The Quantum Computer working with the waves of our NEMF also allows three-dimensional holographic vision through the mind [3], allows telepathic connection [3], and full intuitive creativity [4]. Hypnosis, which puts the conscious to sleep to allow access to the subconscious, surprisingly found that in a state of hypnosis people could calculate 10,000 times faster [5]. This means that we have in the subconscious a Quantum Super-computer, but we are not 
consciously aware of its existence. Our NEMF has the shape of a torus (Figure 1) [6]. The six alternating vortices and anti-vortices of this NEMF are along the backbone of our body, which is axis of spinning of the torus. The six alternating vortices and anti-vortices rule the six endocrinal glands and through them NEMF rules and regulates everything in the body. The torus shape of our NEMF and its dynamic equilibrium is what gives us opportunity to interpret in a new way the classical acupuncture law of five elements reflecting the order in which our organs become active.

\section{The ancient law of five elements}

Specific acupuncture meridian represents each organ on the surface of the skin. Ancient acupuncture [7] considers two types of organs: hollow organs and solid organs.

a. Hollow organs are: stomach, bladder, gall bladder, large intestines, and small intestines. Being hollow the hollow organs are more active and since "Yang" means "active" the hollow organs are Yang organs. They are represented on the surface of the skin by Yang meridians, which run on the back of the body and outer (lateral) sides of arms and legs.

b. Solid organs are: heart, lungs, liver, spleen, and kidneys. Being solid the solid organs are less active and since "Yin" means "passive", the solid organs are Yin organs. They are represented on the surface of the skin by Yin meridians, which run in the front of the body and the inner (medial) side of arms and legs.

The governing meridian (GV) is running vertically downward at the center of the back and head; it is a Yang meridian. The meridian of conception (CV) is running upward vertically at the center of the chest and abdomen. It is a Yin meridian [8]. GV and CV are coupled organs and so are all other organs. Each couple of organs consists of one Yang (hollow) organ and one Yin (solid) organ and usually the organs are closely functionally related. The coupled organs are:

I. Liver - Gall Bladder (LV - GB)

II. Spleen - Stomach (SP - ST)

III. Heart - Small Intestines (HT - SI)

IV. Lungs - Large Intestines (LU - LI)

V. Kidney - Bladder (KI - BL).

The energy Chi runs in the body in the order the organs are listed.

a. From midnight to dawn the Liver is most active.

b. From dawn to noon the Heart is most active.

c. From noon to evening the lungs are most active.

d. From evening to midnight the kidneys are most active.

Usually in the ancient books [7] a pentagram was used to represent the cycle of organ activity. However, since the ancient books state that the spleen is in the center, in some newer books tridimensional octahedral form was used, which includes the Earth (SP - ST) below, the Heaven (GV - CV) up; the four couples of organs are on the horizontal plane in-between.

\section{The new interpretation of the law of five elements}

The torus shape of our NEMF and its dynamic can be represented by two intersected pyramids inscribed in a sphere - one with top up and the other with top down - intersecting at a distance twothirds from the top of the upper pyramid. The two pyramids are in dynamic equilibrium, i.e. pressing more or less on each other. Then the area secluded between the sphere and the intersecting parts of the two pyramids would represent the torus shape form of our NEMF (Figure 2). The form of Fig. 2 is called Tetragrammaton. It can be found in ancient books, like Zohar [9], as the form of the Creator God, who created everything in his image [10].

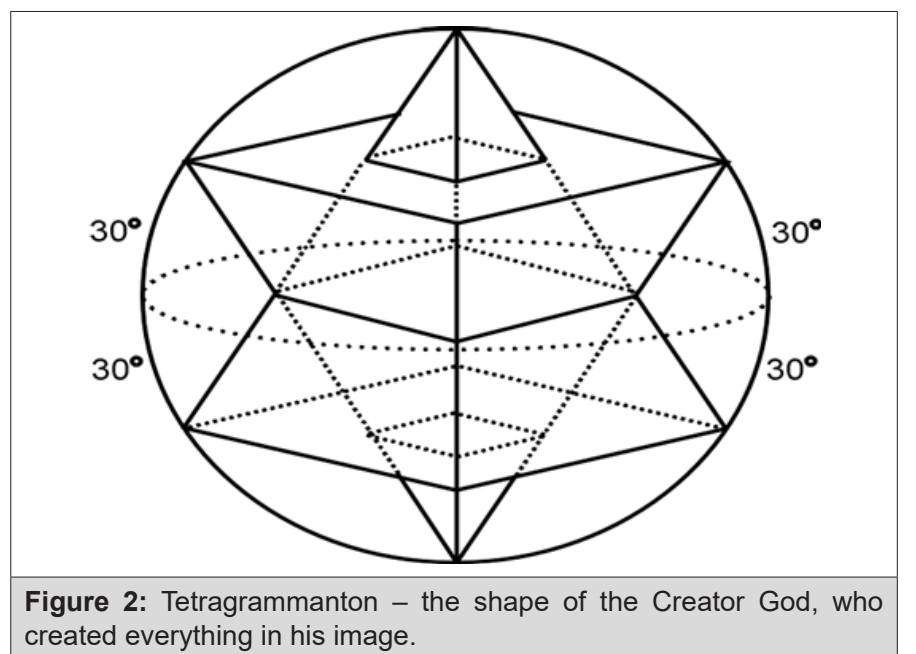

In the ancient texts [9], the pyramid with top down is the Light pyramid. Since "Light" is "Yang", the pyramid with top down is the Yang pyramid. In the ancient texts [8], the pyramid with top up is the Dark (Malhut) pyramid. Since "Darkness" is "Yin", the pyramid with top up is the Yin pyramid. If Tetragrammaton is the shape of all material creations including Man [10], let us consider our NEMF, which rules and regulates everything in the body, as a result of the dynamic equilibrium of two pyramids - a Light pyramid with top down, which represents the Yang (hollow) organs and Dark upright pyramid, which represents the Yin (solid) organs of the body. (Figure 2). Thus, the upside down Light (Yang) pyramid represents the 5 hollow (Yang) organs - with Stomach at the bottom of the pyramid and the four other hollow (Yang) organs at the top. The upright Dark (Yin) pyramid represents the 5 Solid (Yin) organs with CV at the top of the pyramid and the four other Solid (Yin) organs at the bottom four corners. Then the organs on the vertical lines connecting the four corners of the two pyramids would be the coupled Yang-Yin organs with strong functional dependencies, whose meridians form closed circles. The last pair of coupled organs will be the pair of the top (CV) of the upright Dark (Yin) pyramid (CV runs upward in front of the body) and the (GV) of the 
upside-down Light (Yang) pyramids (GV runs downward on the back of the body forming a closed circle with CV). This closed circle represents the couple $\mathrm{CV}-\mathrm{GV}$.

\section{Conclusion}

This article offers a new interpretation of the acupuncture law of five elements, which consider the torus shape of the human NEMF, through which acupuncture cures. Acupuncture cures by correcting the regulating mechanisms, which rule and regulate all the organs in the body through the waves of our NEMF. Our Quantum Computer, which works with the waves of our NEMF, is located in the subconscious, and we don't have conscious awareness that it exists. However, from the subconscious this Quantum Computer rules and regulates the function of all organs. Considering the torus shape of our NEMF, we offered a new presentation of the ancient law of five element used in acupuncture practice.

\section{References}

1. Kuman M (1983) $8^{\text {th }}$ World Congress of Acupuncture, Sofia, Bulgaria.

2. Eory A (1984) Electro-Therapeutic Research. 9: 217-223.

3. Kuman M (2018) Research in Medical and Engineering Sciences 5(3).

4. Kuman M (2018) MOJ Proteomic and Bioinformatics 7(5).

5. Kuman M (2019) Global Journal of Medical Research (K)18(1).

6. Kuman M (2019) Current Trends in Biological and Engineering Sciences 18(1).

7. Veith I (1972) Huang Ti Nei Ching Sue Wen, Berkley-Los Angeles-London.

8. Mann F (1971) Acupuncture - the Ancient Chinese Art of Healing, Cambridge, England.

9. Zohar 23 volumes compiled by Berg Michael.

10. Kuman M (2019) How the Material World Was Created? Origin of its NEMF, Open Access Journal of Mathematical and Theoretical Physics. 2(2): 34-38. 